\title{
Nachweis einer Myoglobinurie
}

H. -W. Schiwara, H. Dräger und A. Heitmann

Gemeinschaftslabor Dr. Loll, Dr. Schiwara, Dr. v. Winterfeld, Bremen

Zusammenfassung:

Folien-Elektrophorese, Gelfiltration, Gegenstromelektrophorese, Doppelimmundiffusion und radiale Immundiffusion wurden auf ihre Eignung zum Nachweis einer Myoglobinurie geprüft.

Schlüsse/wörter:

Myoglobin im Harn - Nachweismethoden

Summary:

Cellulose acetate electrophoresis, gel filtration, counter immunelektrophoresis, double immunodiffusion and radial immunodiffusion are tested for their suitability for the determination of myoglobin in urine.

Keywords:

Myoglobin in urine - methods for determination

Obwohl es zahlreiche Ursachen für eine Myoglobinurie gibt (Muskeltraumen, Herzinfarkt, starke körperliche Belastung von Untrainierten und Myopathien), spielt die Bestimmung von Myoglobin in der klinisch-chemischen Diagnostik eine untergeordnete Rolle. Dies mag daran liegen, daß bei einem Polytraumatisierten die Myoglobinurie im allgemeinen ein Nebenbefund ist. Bei Herzinfarktpatienten wird keineswegs regelmäßig vermehrt Myoglobin im Harn ausgeschieden (1). Bei Muskelerkrankungen dürfte die Enzymdagnostik empfindlicher und spezifischer sein.

Die beiden Hämoproteine Hämoglobin und Myoglobin besitzen als gemeinsame Eigenschaft Pseudoperoxidaseaktivität, $d$. h. sie können aus Peroxid ein Sauerstoffatom auf ein geeignetes Chromogen übertragen. Sie unterscheiden sich durch ihr Molekulargewicht (Hämoglobin: 64500 , Myoglobin: $17200)$. In typischen Fällen kann ohne größeren Laboraufwand die Verdachtsdiagnose „Myoglobinurie" gestellt werden, da bei ausgeprägter Myoglobinurie der Urin lackfarben rot, der Sangur-Test positiv und das Plasma wegen der quantitativen glomerulären Filtration des niedermolekularen Myoglobins normal gefärbt ist. Eine Hämoglobinurie weist dagegen bei gleichem Urinbefund ein hämolytisches Plasma auf (2). Zur genauen Abklärung müssen qualifizierte proteinchemische und immunologische Verfahren eingesetzt werden $(3,4,5)$.

\section{Methoden}

\section{Folien-Elektrophorese}

Die Trennung von Myoglobin und Hämoglobin erfolgt unter den Bedingungen der Hämoglobinelektrophorese auf-Celluloseacetatfolie (6).

Kathodenpuffer: $5,15 \mathrm{~g}$ Barbital-Natrium; 0,92 g Barbital in $1000 \mathrm{ml}$ dest. Wasser lösen; pH 8,6.

Anodenpuffer: $25,2 \mathrm{~g}$ Tris-(hydroximethyl) -aminomethan; $2,5 \mathrm{~g}$ Äthylendiamintetraessigsäure Tetranatriumsalz; $1,9 \mathrm{~g}$ Borsäure in $1000 \mathrm{ml}$ dest. Wasser lösen; $\mathrm{pH}$ a,1.

Die Folie wird mit einer $1+1-$ Mischung aus beiden Puffern getränkt. Ca. $1 \mu$ Probe, Hämoglobin- und Myoglobinvergleichslösung (Konzentration: jeweils ca. $50 \mathrm{mg} / \mathrm{dl}$ ) werden auf der Mitte der Folie 
Abb. 1:

Elektrophoretische Trennung von Hämoglobin und Myoglobin auf Celluloseacetatfolie. a) Hämoglobin; b) und d) Harn eines Patienten mit Myoglobinurie; c) Myoglobin vom Pferd; e) Gemisch von Hämoglobin und Myoglobin vom Pferd

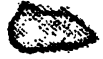

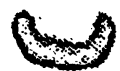

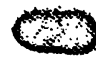

b

C

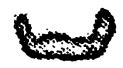

d

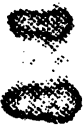

aufgetragen. Falls kein Human-Myoglobin zur Verfügung steht, kann auch Myoglobin vom Pferd (SERVA, Heidelberg) verwendet werden, da es in der Elektrophorese ähnlich wie Human-Myoglobin wandert. Nach 90 Minuten Elektrophorese bei 12,5 V/cm werden Myoglobin und Hämoglobin mit einem Benzidin- bzw. o-Tolidin-Reagenz angefärbt.

Benzidin-Reagenz: $2 \mathrm{~g}$ Benzidin in $20 \mathrm{ml}$ Eisessig unter Erwärmen lösen, abkühlen, auf $100 \mathrm{ml}$ mit dest. Wasser auffüllen und 5 Tropfen 5\%iges Perhydrol zugeben.

o-Tolidin-Reagenz: $0,5 \mathrm{~g}$ o-Tolidin in $100 \mathrm{ml}$ $50 \%$ iger Essigsäure lösen; 3,0 ml 30\%iges Perhydrol zugeben.

Tab. 1:

\section{Methoden zum Nachweis einer Myoglobinurie}

\begin{tabular}{|c|c|c|c|c|}
\hline Methode & $\begin{array}{l}\text { Nachweis- } \\
\text { grenze } \\
\text { (mg/dl) }\end{array}$ & $\begin{array}{l}\text { Untersuchun } \\
\text { dauer (Std.) } \\
\text { mini- } \\
\text { mal }\end{array}$ & $\begin{array}{l}\text { gs- } \\
\text { maxi- } \\
\text { mal }\end{array}$ & $\begin{array}{l}\text { Reagenzien- } \\
\text { kosten } \\
\text { für eine } \\
\text { Einzelanalyse } \\
\text { (DM) }\end{array}$ \\
\hline $\begin{array}{l}\text { 1. Folien- } \\
\text { elektro- } \\
\text { phorese }\end{array}$ & ca. 10 & 2 & & ca. 3,- \\
\hline 2. Gelfiltration & ca. 10 & $3-4$ & & \\
\hline $\begin{array}{l}\text { 3. Gegenstrom- } \\
\text { elektro- } \\
\text { phorese }\end{array}$ & ca. 0,1 & $\left.1^{*}\right)$ & 48 & ca. $4,-$ \\
\hline $\begin{array}{l}\text { 4. Doppel- } \\
\text { immun- } \\
\text { diffusion }\end{array}$ & ca. 0,5 & $\left.12^{\circ}\right)$ & 60 & ca. 2,- \\
\hline $\begin{array}{l}\text { 5. Einfache } \\
\text { radiale } \\
\text { Immun- } \\
\text { diffusion }\end{array}$ & ca. 0,2 & $\left.12^{\circ}\right)$ & 48 & ca. 7,- \\
\hline
\end{tabular}

-) Bei Myoglobinkonzentrationen von über $1 \mathrm{mg} / \mathrm{dl}$
Abb. 2:

Trennung von Hämoglobin und Myoglobin durch Gelfiltration.

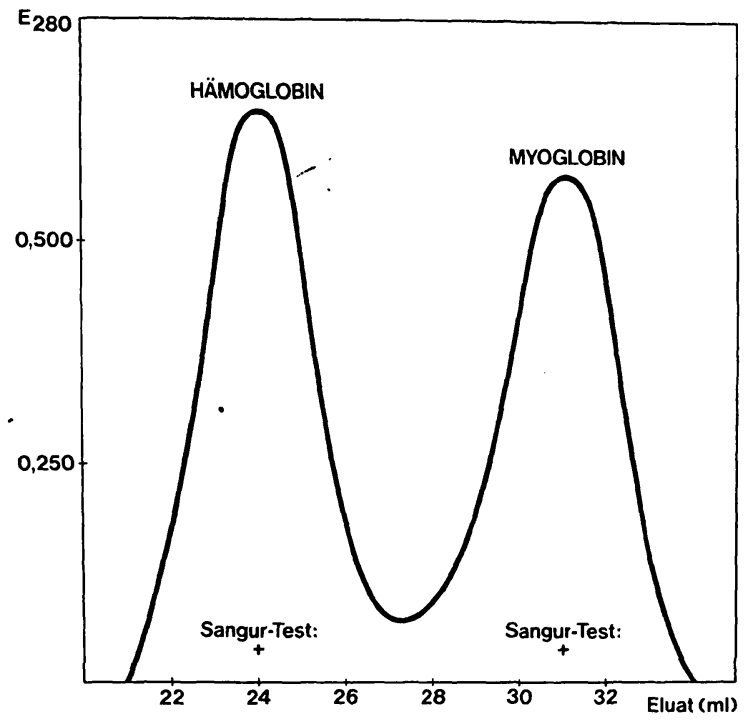

\section{Gelfiltration}

$0,5 \mathrm{ml}$ Probe werden auf eine Sephadex G 75 Säule $(25 \times 250 \mathrm{~mm})$ gegeben und mit phosphatgepufferter $\mathrm{NaCl}$-Lösung pH eluiert. $1 \mathrm{ml}$-Fraktionen werden gesammelt. Die Identifizierung des Hämoglobin- und Myoglobin-Peaks erfolgt durch Registrierung der Extinktion des Eluats bei $280 \mathrm{~nm}$ und durch die positive Reaktion mit dem Sangur-Test. Als Marker kann der Probe ca. 0,5 mg Hämoglobin zugesetzt werden.

\section{Doppelimmundiffusion}

Es wird ein 1,5\%iger Agar in 0,05 $\mathrm{M}$ MichaelisPuffer $\mathrm{pH} \mathrm{8,6}$ verwendet. Die rosettenförmig angeordneten Stanzlöcher haben einen Abstand von $8 \mathrm{~mm}$ und einen Durchmeser von $1,7 \mathrm{~mm}$. Nach Einfüllen von $10 \mu \mathrm{l}$ Antiserum gegen HumanMyoglobin (Behringwerke, Marburg) in das mittlere Stanzloch folgt eine Vordiffusion von 90 Minuten. $10 \mu \mathrm{l}$ Probe werden dann in die äußeren Stanzlöcher gegeben. Die Immundiffusion wird über Nacht bei $37^{\circ} \mathrm{C}$ in feuchter Kammer durchgeführt. Schwache Immunpräzipitate werden nach Wässern (24 Stunden in physiologischer $\mathrm{NaCl}$-Lösung) und Trocknen des Agars unter Filtrierpapier mit Amidoschwarz angefärbt. 


\section{Abb. 3:}

Nachweis von Myoglobin mit der Gegenstromelektrophorese (obere Bildhälfte) und Doppelimmundiffusion (untere Bildhälfte). Die beiden Rosetten sind identisch. Jeweils in der Mitte wurde das Antimyoglobinserum, rechts myoglobin- und links hämoglobinhaltiger Harn eingefüllt.

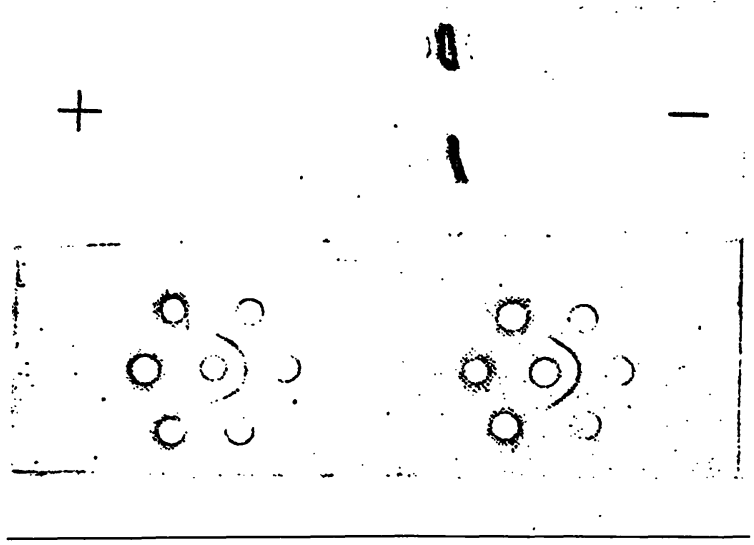

\section{Gegenstrom-Elektrophorese}

In einen 1,5\%igen Agar in 0,05 M Michaelis-Puffer pH 8,6 werden im Abstand von $4 \mathrm{~mm}$ Löcher gestanzt (Durchmesser: $4 \mathrm{~mm}$ ). In das kathodisch gelegene Stanzloch werden $20 \mu$ l Urin eingefüllt. Nach 30 Minuten Elektrophorese bei $10 \mathrm{~V} / \mathrm{cm}(0,1 \mathrm{M} \mathrm{Mi}$ chaelis-Puffer $\mathrm{pH} 8,6$ ) werden in das anodisch gelegene Stanzloch $20 \mu \mathrm{l}$ Antiserum pipettiert. Die Elektrophorese wird 30 Minuten fortgeführt. Schwache Immunpräzipitate werden nach Wässern (24 Stunden in physiologischer $\mathrm{NaCl}$-Lösung) und Trocknen des Agars unter Filtrierpapier mit Amidoschwarz angefärbt.

\section{Einfache radiale Immundiffusion}

Die einfache radiale Immundiffusion wird in 1,5\%iger Agarose mit 15\% Antiserum gegen $\mathrm{Hu}-$ man-Myoglobin (Behringwerke, Marburg) durchgeführt. Zu $4,75 \mathrm{ml}$ auf ca. $60^{\circ} \mathrm{C}$ abgekühlte Agaroselösung (1,5\% Agarose in 0,1 $\mathrm{M}$ Michaelis-Puffer $\mathrm{pH}$ 8,6; Natriumazid $1 \mathrm{mg} / \mathrm{ml}$ ) wird $0,75 \mathrm{ml}$ Antimyoglobinserum gemischt. Die Antikörper-Agarose-Lösung wird in eine für die LC-PartigenPlatten (Behringwerke, Marburg) verwendete Kunststoffschale gegossen. Nach dem Erstarren des Agars werden 12 Löcher ausgestanzt (Durchmesser $4 \mathrm{~mm}$ ) $.2 \times 10 \mu \mathrm{l}$ Probe bzw. Standard werden in die Stanzlöcher eingefüllt. Bei stark positivem SangurTest wird außerdem die Probe zur Vermeidung eines
Abb. 4:

Eichkurve für die Myoglobinbestimmung mit der einfachen radialen Immundiffusion.

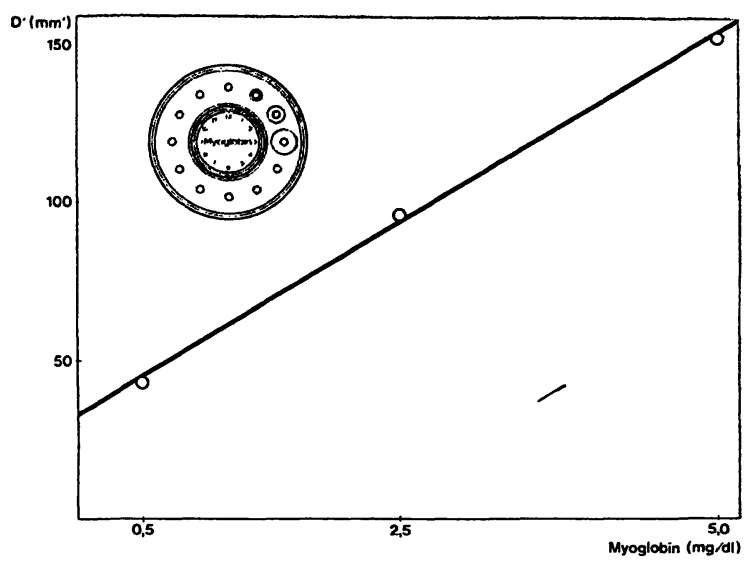

Prozonenphänomens 1:10 verdünnt angesetzt. Nach 48 Stunden werden die Präzipitate ausgemessen.

Im Handel ist bisher kein für die radiale Immundiffusion geeignetes Standard-Präparat erhältlich. Für Versuchszwecke stellten uns freundlicherweise die Behringwerke ein Humanmyoglobin-Präparat zur Verfügung. Eigene Standardpräparate wurden aus myoglobinhaltigen Urinen hergestellt: $1 \mathrm{ml}$ des etwa 10-fach konzentrierten Urins (Diaflo Ultrafiltrationsmembranen UM 10 der Firma Amicon, Witten) wird auf eine Sephadex G 75 Säule gegeben und mit phosphatgepufferter Kochsalzlösung ph 7,2 eluiert. Die myoglobinhaltigen Fraktionen werden gesammelt und die Myoglobinkonzentration mit der radialen Immundiffusion bestimmt. Die Eichung erfolgt mit dem von den Behringwerken überlassenen Myoglobin-Standard.

Außerdem kann die Myoglobinkonzentration mit einer photometrischen Methode (Oxidation von 0 -Tolidin in Gegenwart von $\mathrm{H}_{2} \mathrm{O}_{2}$ ) gemessen werden (7). Als Standard wird Myoglobin vom Pferd verwendet. Die durch Gelfiltration gereinigten Präparate werden durch Zugabe von Albumin (100 $\mathrm{mg} / \mathrm{ml})$ und Natriumazid $(1 \mathrm{mg} / \mathrm{ml})$ stabilisiert (8). Die Lagerung erfolgt bei $+4^{\circ} \mathrm{C}$ bzw. $-20^{d} \mathrm{C}$.

\section{Diskussion}

Unsere Erfahrungen mit unterschiedlichen Nach- 
weismethoden von Myoglobin möchten wir kurz zusammenfassen.

Bevor eines der beschriebenen Verfahren eingesetzt wird, sollte mit dem Sangur-Test geprüft werden, ob überhaupt eine pathologische Hämo- oder Myoglobinurie vorliegt. Bei negativem Sangur-Test ist eine pathologische Myoglobinurie praktisch ausgeschlossen, da mit dem Teststreifen noch $0,05 \mathrm{mg} / \mathrm{dl}$ Myoglobin nachgewiesen werden kann und die physiologische Myoglobinurie unter $0,03 \mathrm{mg} / \mathrm{dl}$ liegt (9).

Die untersuchten Methoden sind in Tabelle $1 \mathrm{ge}-$ genübergestellt. Elektrophorese und Gelfiltration ermöglichen den gleichzeitigen Nachweis von Hämoglobin und Myoglobin, sofern die beiden Hämoproteine in ausreichender Konzentration ausgeschieden werden. Mit den immunologischen Methoden kann nur Myoglobin identifiziert werden. Die drei Verfahren haben etwa die gleiche Empfindlichkeit. Besonders schnell gelingt mit der Gegenstromelek-

Schrifttum:

1. KOLENDORF, $K$, PEDERSEN, F., CHRISTIANSEN, E., GAD, I.: Rocket immuno1. KOLENDOs. elektrophoresis of myoglobin in urine in
Scand. (Suppl.) 623.103-107 (1978).

2. SPAET, T. H., ROSENTHAL. M. D., DAMESHEK. W.: Idiopathic myoglobinuria in man: report of a case. Blood 9,881 (1954).

3. ROSS-FANELLI, A. ANTONINI. E., Heterogeneity of Human Myoglobin. Arch. Biochem. Biophys. 65, 587-590 (1956).

4. BERMAN, M. C. KENCH. I. E.: Seperation of myoglobin and haemoglobin on a 4. BERMAN, M. C.. KENCH. I. E.: Seperation of myog dextran gel. J. Clin. Pathol. 16, 385 (1963). trophorese der Nachweis einer Myoglobinurie. Die Doppelimmundiffusion zeichnet sich durch ihre Wirtschaftlichkeit aus. Am geeignetsten für das Routinelabor ist die einfache radiale Immundiffusion. Quantitative Messungen sind möglich, wenn ein entsprechendes Standardpräparat zur Verfügung steht. Allerdings ist nach unseren Erfahrungen entgegen den Angaben in der Literatur $(5,8)$ die Empfindlichkeit der Methode nicht ausreichend, um auch geringe, vermuttich klinisch irrelevante Myoglobinurien (zwischen 0,03 und 0,2 mg/dl) zu erfassen. Versuche durch Konzentrierung der Proben mit Kollodiumhülsen SM 13200 (Sartorius, Göttingen), Myoglobin auch in diesem Bereich mit der radialen Immundiffusion messen zu können, waren leider nicht erfolgreich. Die Analyse so niedriger - Myoglobinkonzentrationen ist vermutlich nur mit einem Radioimmunoassay möglich. Ein im Handel befindlicher Kit (Nuclear Medical Systems Inc., Newport Beach) ist allerdings nur für die Bestimmung von Myoglobin im Serum ausgelegt.

5. KELLER, P., MÜNSCHER, G., PFEIFFER, G.: Immunologischer Myoglobinnachweis beim Herzinfakt. Therapiewoche 26, 1784-1793 (1976).

6. KOHN, I.: Zur Technik der Celluloseazetat-Elektrophorese. Arztl. Lab. 10. 269-278 (1964).

7. HENRY, R. J., CANNON, O. C., WINKELMANN, I. W.: Clinical Chemistry. Principles and Technics. New York 1974, S. 1141

and Technics. New York BOESKEN, S., MAMIER, A.: Human Myoglobin: Preparation. 8. BOESKEN, W. H.. BOESKEN, S., MAMIER, A.: Human Myoglobin:
Quantitation and Standardization. Res. exp. Med. 171, 71-78 (1977).

9. KUTTER, D., VAN OUDHEUSDEN, A. P. M., HILVERS, A. G., NECHVILE, K., VAN BUUL, T. KOLLER, P. U.: Die Brauchbarkeit eines neuen Teststreifens zum Nachweis von Erythrocyten und Hämoglobin im Harn, Dtsch. Med. Wschr. 99, 2332-2335 (1974).

Anschrift des Verfassers:

Dr. med.. Hans-Walter Schiwara

Straßburger Str. 19

D-2800 Bremen 1 